\title{
Mathematical Model for Lassa Fever Transmission and Control
}

\author{
Anorue Onyinyechi Favour ${ }^{1, *}$, Okeke Anthony Anya ${ }^{2}$ \\ ${ }^{1}$ Department of Mathematics, Michael Okpara University of Agriculture Umudike Abia-State, Umuahia, Abia-State, Nigeria \\ ${ }^{2}$ Department of Mathematics, Federal University Gashua, Gashua, Yobe-State, Nigeria
}

Email address:

onyinyechianorue@gmail.com (A. O. Favour)

*Corresponding author

To cite this article:

Anorue Onyinyechi Favour, Okeke Anthony Anya. Mathematical Model for Lassa Fever Transmission and Control. Mathematics and Computer Science. Vol. 5, No. 6, 2020, pp. 110-118. doi: 10.11648/j.mcs.20200506.13

Received: October 15, 2020; Accepted: October 28, 2020; Published: December 16, 2020

\begin{abstract}
Lassa fever is an acute hemorrhagic zoonotic illness (possible transmission from infected animals to humans), caused by Lassa virus whose reservoir host is the Mastomys natalensis (Rodent). It is a disease with a duration of 2-21 days that strives more in African nations and countries with poor water and environmental sanitation. In this paper, a deterministic model for Lassa fever is formulated buttressing the various stages of infection of the disease. We studied the existence and uniqueness of the solutions. The steady states of the model are determined and the basic reproduction number is analyzed with a threshold parameter $R_{0}$ which shows persistence of the disease if and only if $R_{0}>1$ using the next generation matrix. The treatment strategies considered amidst others are the use of antiviral drug and to quarantine infected individuals on early diagnosis of the infection on the asymptomatic and symptomatic individuals respectively. Numerically, it was evidential that the quarantine system has a great positive effect on the rate of recovery of the infected individuals and also in curbing the risk of infection in the environment which can help safeguard the population. A relapse on this method will lead to reinfection of the disease thereby bringing the population to a point of danger.
\end{abstract}

Keywords: Lassa Fever, Infection Process, Quarantine, Equilibrium, Stability

\section{Introduction}

Lassa fever (LF) which is caused by Lassa virus (LASV) was first discovered in Nigeria in 1969 [1], an acute viral hemorrhagic illness, a single stranded RNA virus belonging to the family Arena viridae whose reservoir is the Rodent: the multi-mammate Rat (Mastomys natalensis). It is mostly found in Sub-Saharan regions of Africa, the West African Countries like Liberia, Sierra Leone, Guinea and especially Nigeria who has been under its attack for the past seven decades [2]. The infection of the virus to human occurs by direct or indirect exposure to animal excrement through the respiratory or gastro intestinal tracts or eating contaminated food, touching soiled objects or exposure to open cuts or sores $[5,6,10]$. Signs and symptoms of Lassa fever typically occur 1-3 weeks after the patient comes in contact with the virus. Majority of Lassa fever virus infections show mild and undiagnosed symptoms which include slight fever, general weakness and headache while the serious cases progress to more severe signs like vomiting facial swellings pain in the chest, back abdomen and shock degenerating to hemorrhage (bleeding in gums, eyes or nose), thus showing symptomatic and asymptomatic signs $[8,9,19]$. Lassa fever has also been found to have some links with neurological problems which include hearing loss, which may be transient or permanent [7, 18]. It has been found that the disease has a high rate of transmission during sexual intercourse, the virus can be present in urine for between three and nine weeks after infection and it can be transmitted in semen for up to three months after becoming infected $[3,4,15]$. Lassa fever can be treated with antiviral drug ribavirin which has been very effective when given early in the course of the disease [19].

Various theoretical studies have been carried out on mathematical modeling of Lassa fever transmission dynamics, focusing on a number of different issues. [11-14], but not many focus on the symptomatic and asymptomatic 
behavior of Lassa fever and the possibility of transferring it through sexual intercourse. We present a simple model with the goal of showing the effect of symptomatic and asymptomatic nature of the virus and the effect of quarantining in curbing the spread of the disease.

\section{Model Formulation}

We formulate a deterministic model for the spread dynamics and control of Lassa fever that considers human and vector population at a given time $(\mathrm{t})$. The human population is divided into six compartments: susceptible humans $\left(\mathrm{S}_{\mathrm{H}}\right)$, exposed humans $\left(\mathrm{E}_{\mathrm{H}}\right)$, infectious asymptomatic humans $\left(\mathrm{I}_{\mathrm{HA}}\right)$, infectious symptomatic humans $\left(\mathrm{I}_{\mathrm{HS}}\right)$, quarantine humans $\left(\mathrm{I}_{\mathrm{HQ}}\right)$, and the recovered humans $\left(\mathrm{R}_{\mathrm{H}}\right)$, the total population of humans $\mathrm{N}_{\mathrm{H}}(\mathrm{t})$ is given by:

$$
\mathrm{N}_{\mathrm{H}}=\mathrm{S}_{\mathrm{H}}+\mathrm{E}_{\mathrm{H}}+\mathrm{I}_{\mathrm{HA}}+\mathrm{I}_{\mathrm{HS}}+\mathrm{I}_{\mathrm{HQ}}+\mathrm{R}_{\mathrm{H}} \text {. }
$$

On the other hand, vector population is divided into three compartments: susceptible vectors $\left(\mathrm{S}_{\mathrm{V}}\right)$, exposed vectors $\left(\mathrm{E}_{\mathrm{V}}\right)$, and the infectious vectors $\left(\mathrm{I}_{\mathrm{V}}\right)$. The total population of vectors $\mathrm{N}_{\mathrm{V}}(\mathrm{t})$ is given by:

$$
\mathrm{N}_{\mathrm{V}}=\mathrm{S}_{\mathrm{V}}+\mathrm{E}_{\mathrm{V}}+\mathrm{I}_{\mathrm{V}}
$$

The model parameters and their descriptions: Birth or immigration rate of humans into the susceptible human population $\wedge_{\mathrm{H}}$, Birth or immigration rate of vectors into the susceptible vector population $\Lambda_{\mathrm{H}}$, Natural death rate of humans and vectors through which they exist from their individual populations, $\mu_{\mathrm{H}}$ and $\mu_{\mathrm{V}}$ respectively. The disease induced death rate of human population of asymptomatic infectious humans and symptomatic infectious humans $\omega_{1}$ and $\omega_{2}$ respectively, effective contact rate between asymptomatic infectious humans, symptomatic infectious humans, quarantine humans and infectious vector or its contaminants with the susceptible human are $\beta_{1} \mathrm{I}_{\mathrm{HA}} \mathrm{S}_{\mathrm{H}}, \beta_{2} \mathrm{I}_{\mathrm{HS}} \mathrm{S}_{\mathrm{H}}, \beta_{3} \mathrm{I}_{\mathrm{HQ}} \mathrm{S}_{\mathrm{H}}$ and $\beta_{4} \mathrm{I}_{\mathrm{V}} \mathrm{S}_{\mathrm{H}}$ respectively, of which $\beta_{\mathrm{H}}=\beta_{1} \mathrm{I}_{\mathrm{HA}} \mathrm{S}_{\mathrm{H}}, \beta_{2} \mathrm{I}_{\mathrm{HS}} \mathrm{S}_{\mathrm{H}}, \beta_{3} \mathrm{I}_{\mathrm{HQ}} \mathrm{S}_{\mathrm{H}}$ and $\beta_{4} \mathrm{I}_{\mathrm{V}} \mathrm{S}_{\mathrm{H}}$. Effective contact rate between infectious vector and susceptible vector $\beta_{4} I_{V} S_{V}$. Rate at which the exposed vectors move into the infectious population $\gamma_{\mathrm{V}}$, recovery rate of the quarantine humans $\eta$, proportion of exposed that are asymptomatic $a \alpha$, proportion of the exposed that are symptomatic $(1-\mathrm{a}) \alpha$, latency period of Lassa fever in humans $\alpha^{-1}$ and human infectious period $\delta$.

\subsection{Assumptions}

1). There is homogeneous mixing of members of the population under consideration.

2). Due to the yearly outbreaks of Lassa fever, there might be new births or inflow of susceptible individuals as well as natural deaths permitting a demographic process to take place.

$3)$. Infection is passed on through vector contaminants in the environment or through interaction with infectious humans.

4). Individuals move to R only from $I_{\mathrm{HQ}}$ due to permanent immunity confer on them as a result of effective treatment.
Table 1. Description of variables.

\begin{tabular}{ll}
\hline $\mathrm{S}_{\mathrm{H}}$ & Total number of susceptible population. \\
$E_{H}$ & Total number of exposed population. \\
$\mathrm{I}_{\mathrm{HA}}$ & Total number of infectious asymptomatic population. \\
$\mathrm{I}_{\mathrm{HS}}$ & Total number of infectious symptomatic population. \\
$\mathrm{I}_{\mathrm{HO}}$ & Total number of quarantine population. \\
$\mathrm{R}_{\mathrm{H}}$ & Total number of recovered population. \\
$\mathrm{S}_{\mathrm{V}}$ & Total number of, susceptible vector population. \\
$\mathrm{E}_{\mathrm{V}}$ & Total number of exposed vector population. \\
$\mathrm{I}_{\mathrm{V}}$ & Total number of infectious vector population. \\
\hline
\end{tabular}

\subsection{Model Equations}

The transmissions between model classes can be expressed by the following system of first order differential equations:

$$
\begin{aligned}
& \frac{d S_{H}}{d t}=\Lambda_{H}-\beta_{1} I_{H A} S_{H}-\beta_{2} I_{H S} S_{H}-\beta_{3} I_{H Q} S_{H}-\beta_{4} I_{V} S_{H}-\mu_{H}(1) \\
& \frac{d E_{H}}{d t}=\beta_{1} I_{H A} S_{H}+\beta_{2} I_{H S} S_{H}+\beta_{3} I_{H Q} S_{H}+\beta_{4} I_{V} S_{H}-\left(\alpha+\mu_{H}\right) E_{H} \\
& \frac{\mathrm{dI}_{\mathrm{HA}}}{\mathrm{dt}}=\mathrm{a} \alpha \mathrm{E}_{\mathrm{H}}-\left(\delta+\mu_{\mathrm{H}}+\omega_{1}\right) \mathrm{I}_{\mathrm{HA}} \\
& \frac{\mathrm{dI}_{\mathrm{HS}}}{\mathrm{dt}}=(\mathrm{I}-\mathrm{a}) \alpha \mathrm{E}_{\mathrm{H}}-\left(\delta+\mu_{\mathrm{H}}+\omega_{2}\right) \mathrm{I}_{\mathrm{HS}} \\
& \frac{\mathrm{dI}_{\mathrm{HQ}}}{\mathrm{dt}}=\left(\mathrm{I}_{\mathrm{HA}}+\mathrm{I}_{\mathrm{HS}}\right) \delta-\left(\mu_{\mathrm{H}}+\eta\right) \mathrm{I}_{\mathrm{HQ}} \\
& \frac{\mathrm{dR}_{\mathrm{H}}}{\mathrm{dt}}=\eta \mathrm{I}_{\mathrm{HQ}}-\mu_{\mathrm{H}} \mathrm{R}_{\mathrm{H}} \\
& \frac{d S_{V}}{d t}=\Lambda_{V}-\left(\beta_{4} I_{V}+\mu_{V}+\varphi_{V}\right) S_{V} \\
& \frac{d E_{V}}{d t}=\beta_{4} I_{V} S_{v}-\left(\gamma_{V}+\mu_{V}+\varphi_{V}\right) E_{v} \\
& \frac{\mathrm{dI}_{\mathrm{V}}}{\mathrm{dt}}=\gamma_{\mathrm{V}} \mathrm{E}_{\mathrm{V}}-\left(\mu_{\mathrm{V}}+\varphi_{\mathrm{V}}\right) \mathrm{I}_{\mathrm{V}}
\end{aligned}
$$

With the initial conditions $\mathrm{S}_{\mathrm{H}}(0)=\mathrm{S}_{\mathrm{H}}^{0}, \mathrm{E}_{\mathrm{H}}(0)=$ $\mathrm{E}_{\mathrm{H}}^{0}, \mathrm{I}_{\mathrm{HA}}(0)=\mathrm{I}_{\mathrm{HA}}^{0} \mathrm{I}_{\mathrm{HS}}(0)=\mathrm{I}_{\mathrm{HS}}^{0}, \mathrm{I}_{\mathrm{HQ}}(0)=\mathrm{I}_{\mathrm{HQ}}^{0}, \mathrm{R}_{\mathrm{H}}(0)=$ $\mathrm{R}_{\mathrm{H}}^{0}, \mathrm{~S}_{\mathrm{V}}(0)=\mathrm{S}_{\mathrm{V}}^{0}, \mathrm{E}_{\mathrm{V}}(0)=\mathrm{E}_{\mathrm{V}}^{0}, \mathrm{I}_{\mathrm{V}}(0)=\mathrm{I}_{\mathrm{V}}^{0}$. The total human population $\mathrm{N}_{\mathrm{H}}(\mathrm{t})$ is given by:

$\mathrm{N}_{\mathrm{H}}(\mathrm{t})=\mathrm{S}_{\mathrm{H}}(\mathrm{t})+\mathrm{E}_{\mathrm{H}}(\mathrm{t})+\mathrm{I}_{\mathrm{HA}}(\mathrm{t})+\mathrm{I}_{\mathrm{HS}}(\mathrm{t})+\mathrm{I}_{\mathrm{HQ}}(\mathrm{t})+\mathrm{R}_{\mathrm{H}}(\mathrm{t})$.

And satisfies the ordinary differential equation

$$
\frac{\mathrm{dN}_{\mathrm{H}}}{\mathrm{dt}}=\Lambda_{\mathrm{H}}-\mu_{\mathrm{H}} \mathrm{N}_{\mathrm{H}}-\omega_{1} \mathrm{I}_{\mathrm{HA}}-\omega_{2} \mathrm{I}_{\mathrm{HS}}+\eta \mathrm{I}_{\mathrm{HQ}} .
$$

With the initial condition above, we obtain:

$$
\frac{\mathrm{dN}_{\mathrm{H}}}{\mathrm{dt}} \leq \Lambda_{\mathrm{H}}-\mu_{\mathrm{H}} \mathrm{N}_{\mathrm{H}}
$$

On the other hand, the total population of the vector is given by:

$$
\mathrm{N}_{\mathrm{V}}(\mathrm{t})=\mathrm{S}_{\mathrm{V}}(\mathrm{t})+\mathrm{E}_{\mathrm{V}}(\mathrm{t})+\mathrm{I}_{\mathrm{V}}(\mathrm{t}) .
$$

This also satisfies the ordinary differential equation:

$$
\frac{\mathrm{dN}_{\mathrm{V}}}{\mathrm{dt}} \leq \Lambda_{\mathrm{V}}-\left(\mu_{\mathrm{H}}+\varphi_{\mathrm{V}}\right) \mathrm{N}_{\mathrm{V}}
$$

In this section, we begin the model analysis by showing that all feasible solutions of the model system are uniformly 
bounded in a set of $\Omega$. Thus, the feasible region

$$
\begin{gathered}
\Omega=\left\{\left(\mathrm{S}_{\mathrm{H}}, \mathrm{E}_{\mathrm{H}}, \mathrm{I}_{\mathrm{HA}}, \mathrm{I}_{\mathrm{HS}}, \mathrm{I}_{\mathrm{HQ}}, \mathrm{R}_{\mathrm{H}}, \mathrm{S}_{\mathrm{V}}, \mathrm{E}_{\mathrm{V}}, \mathrm{I}_{\mathrm{V}}\right) \in \mathcal{R}_{+}^{9}: \mathrm{N}_{\mathrm{H}} \leq\right. \\
\left.\frac{\Lambda_{\mathrm{H}}}{\mu_{\mathrm{H}}}, \mathrm{N}_{\mathrm{V}} \leq \frac{\Lambda_{\mathrm{V}}}{\mu_{\mathrm{V}}+\varphi_{\mathrm{V}}}\right\}
\end{gathered}
$$

Therefore, considering the derivatives of the human and vector population (1.10) and (1.11) respectively with proper substitutions and simplifications, we have:

$$
\frac{\mathrm{d}}{\mathrm{dt}}\left(\mathrm{S}_{\mathrm{H}}+\mathrm{E}_{\mathrm{H}}+\mathrm{I}_{\mathrm{HA}}+\mathrm{I}_{\mathrm{HS}}+\mathrm{R}_{\mathrm{H}}\right) \leq \frac{\Lambda_{\mathrm{H}}}{\mu_{\mathrm{H}}},
$$

Therefore

$$
\lim _{\mathrm{t} \rightarrow \infty} \mathrm{N}_{\mathrm{H}}(\mathrm{t}) \leq \frac{\Lambda_{\mathrm{H}}}{\mu_{\mathrm{H}}}
$$

And

$$
\begin{gathered}
\frac{d}{d t}\left(S_{V}+E_{V}+I_{V}\right) \leq \frac{\Lambda_{V}}{\mu_{V}+\varphi_{V}}, \\
\lim _{t \rightarrow \infty} N_{V}(t) \leq \frac{\Lambda_{V}}{\mu_{V}+\varphi_{V}} .
\end{gathered}
$$

Hence, the feasible solution of the model equations (1.1)(1.9) enter the region $\Omega$ which is a positively invariant set. Thus, the system is both mathematically and epidemiologically well posed. Therefore, for an initial starting point $x \in \Omega$, the trajectory of $x$ lies in $\Omega$ and so it is sufficient to restrict our analysis on $\Omega$. So that under the dynamics described by the model equations in (1.1)-(1.9), the closed set $\Omega$ is positively invariant set.

\section{Model Analysis}

\subsection{Existence and Uniqueness of Solutions}

Theorem 2.1[16, 17]

Let $\mathrm{D}^{1}$ denotes the region

$$
\left|t-t_{0}\right| \leq b, x=\left(x_{1}, x_{2}, \ldots, x_{n}\right), x_{0}=\left(x_{10}, x_{20}, \ldots, x_{n 0}\right)
$$

And suppose that $\mathrm{f}(\mathrm{t}, \mathrm{x})$ satisfies the Lipchitz condition

$$
\left\|\mathrm{f}\left(\mathrm{t}, \mathrm{x}_{1}\right)-\left(\mathrm{t}, \mathrm{x}_{2}\right)\right\| \leq \mathrm{k}\left\|\mathrm{x}_{1}-\mathrm{x}_{2}\right\|
$$

Whenever the pairs $\left(\mathrm{t}, \mathrm{x}_{1}\right)$ and $\left(\mathrm{t}, \mathrm{x}_{2}\right)$ belong $D^{1}$, where $\mathrm{k}$ is a positive constant. Then, there is a constant $\delta>0$ such that there exists a unique continuous vector solution $x(t)$ of the system (2.1) in the interval $\left|t-t_{0}\right| \leq \delta$. The condition (2.2) is satisfied by the requirement that $\frac{\partial f_{i}}{\partial x_{j}}, i . j=1,2, \ldots \ldots, n$ be continuous and bounded in $\mathrm{D}^{1}$. Consider the region $1 \leq \mathcal{E} \leq$ $\mathrm{R}$, we check for a bounded solution of the form $0<\mathrm{R}<\infty$.

Theorem 2.2

Let $\mathrm{D}$ denotes the region defined earlier such that we can get both the region and the bounded solution hold, and then there exists a unique solution of the model system (1.1)-(1.9) which is bounded inD.

Proof

Let

$$
\begin{gathered}
\mathrm{f}_{1}=\Lambda_{\mathrm{H}}-\beta_{1} \mathrm{I}_{\mathrm{HA}} \mathrm{S}_{\mathrm{H}}-\beta_{2} \mathrm{I}_{\mathrm{HS}} \mathrm{S}_{\mathrm{H}}-\beta_{3} \mathrm{I}_{\mathrm{HQ}} \mathrm{S}_{\mathrm{H}}-\beta_{4} \mathrm{I}_{\mathrm{V}} \mathrm{S}_{\mathrm{H}}-\mu_{\mathrm{H}} \\
\mathrm{f}_{2}=\beta_{1} \mathrm{I}_{\mathrm{HA}} \mathrm{S}_{\mathrm{H}}+\beta_{2} \mathrm{I}_{\mathrm{HS}} \mathrm{S}_{\mathrm{H}}+\beta_{3} \mathrm{I}_{\mathrm{HQ}} \mathrm{S}_{\mathrm{H}}+\beta_{4} \mathrm{I}_{\mathrm{V}} \mathrm{S}_{\mathrm{H}}-\left(\alpha+\mu_{\mathrm{H}}\right) \mathrm{E}_{\mathrm{H}} \\
\mathrm{f}_{3}=\mathrm{a} \alpha \mathrm{E}_{\mathrm{H}}-\left(\delta+\mu_{\mathrm{H}}+\omega_{1}\right) \mathrm{I}_{\mathrm{HA}} \\
\mathrm{f}_{4}=(\mathrm{I}-\mathrm{a}) \alpha \mathrm{E}_{\mathrm{H}}-\left(\delta+\mu_{\mathrm{H}}+\omega_{2}\right) \mathrm{I}_{\mathrm{HS}} \\
\mathrm{f}_{5}=\left(\mathrm{I}_{\mathrm{HA}}+\mathrm{I}_{\mathrm{HS}}\right) \delta-\left(\mu_{\mathrm{H}}+\eta\right) \mathrm{I}_{\mathrm{HQ}} \\
\mathrm{f}_{6}=\eta \mathrm{I}_{\mathrm{HQ}}-\mu_{\mathrm{H}} \mathrm{R}_{\mathrm{H}} \\
\mathrm{f}_{7}=\Lambda_{\mathrm{V}}-\left(\beta_{4} \mathrm{I}_{\mathrm{V}}+\mu_{\mathrm{V}}+\varphi_{\mathrm{V}}\right) \mathrm{S}_{\mathrm{V}} \\
\mathrm{f}_{8}=\beta_{\mathrm{V}} \mathrm{I}_{\mathrm{V}} \mathrm{S}_{\mathrm{V}}-\left(\gamma_{\mathrm{V}}+\mu_{\mathrm{V}}+\varphi_{\mathrm{V}}\right) \mathrm{E}_{\mathrm{V}} \\
\mathrm{f}_{9}=\gamma_{V} \mathrm{E}_{\mathrm{V}}-\left(\mu_{\mathrm{V}}+\varphi_{\mathrm{V}}\right) \mathrm{I}_{\mathrm{V}}
\end{gathered}
$$

We show that the $\frac{\partial \mathrm{f}_{\mathrm{i}}}{\partial \mathrm{x}_{\mathrm{j}}} \mathrm{i}, \mathrm{j}=1,2, \ldots, 9$ are continuous by considering the partial derivatives below:

$$
\begin{gathered}
\left|\frac{\partial \mathrm{f}_{1}}{\partial \mathrm{S}_{\mathrm{H}}}\right|=\left|-\left(\beta_{1} \mathrm{I}_{\mathrm{HA}}+\beta_{2} \mathrm{I}_{\mathrm{HS}}+\beta_{3} \mathrm{I}_{\mathrm{HQ}}+\beta_{4} \mathrm{I}_{\mathrm{V}}+\mu_{\mathrm{H}}\right)\right|<\infty \\
\left|\frac{\partial \mathrm{f}_{1}}{\partial \mathrm{I}_{\mathrm{V}}}\right|=\left|-\beta_{4} \mathrm{~S}_{\mathrm{H}}\right|<\infty,\left|\frac{\partial \mathrm{f}_{1}}{\partial \mathrm{I}_{\mathrm{HA}}}\right|=\left|-\beta_{1} \mathrm{~S}_{\mathrm{H}}\right|<\infty,\left|\frac{\partial \mathrm{f}_{1}}{\partial \mathrm{I}_{\mathrm{HS}}}\right|=\left|-\beta_{2} \mathrm{~S}_{\mathrm{H}}\right|<\infty,\left|\frac{\partial \mathrm{f}_{1}}{\partial \mathrm{I}_{\mathrm{HQ}}}\right|=\left|-\beta_{3} \mathrm{~S}_{\mathrm{H}}\right|<\infty, \\
\left|\frac{\partial \mathrm{f}_{1}}{\partial \mathrm{R}_{\mathrm{H}}}\right|=\left|\frac{\partial \mathrm{f}_{1}}{\partial \mathrm{S}_{\mathrm{V}}}\right|=\left|\frac{\partial \mathrm{f}_{1}}{\partial \mathrm{E}_{\mathrm{V}}}\right|=\left|\frac{\partial \mathrm{f}_{1}}{\partial \mathrm{E}_{\mathrm{H}}}\right|=0<\infty . \\
\left|\frac{\partial \mathrm{f}_{2}}{\partial \mathrm{S}_{\mathrm{H}}}\right|=\left|\left(\beta_{1} \mathrm{I}_{\mathrm{HA}}+\beta_{2} \mathrm{I}_{\mathrm{HS}}+\beta_{3} \mathrm{I}_{\mathrm{HQ}}+\beta_{4} \mathrm{I}_{\mathrm{V}}+\mu_{\mathrm{H}}\right)\right|,\left|\frac{\partial \mathrm{f}_{2}}{\partial \mathrm{E}_{\mathrm{H}}}\right|=\left|-\left(\alpha+\mu_{\mathrm{H}}\right)\right|<\infty,
\end{gathered}
$$




$$
\begin{aligned}
& \left|\frac{\partial \mathrm{f}_{2}}{\partial \mathrm{I}_{\mathrm{HA}}}\right|=\left|\beta_{1} \mathrm{~S}_{\mathrm{H}}\right|,\left|\frac{\partial \mathrm{f}_{2}}{\partial \mathrm{I}_{\mathrm{HS}}}\right|=\left|\beta_{2} \mathrm{~S}_{\mathrm{H}}\right|,\left|\frac{\partial \mathrm{f}_{2}}{\partial \mathrm{I}_{\mathrm{HQ}}}\right|=\left|\beta_{3} \mathrm{~S}_{\mathrm{H}}\right|,\left|\frac{\partial \mathrm{f}_{2}}{\partial \mathrm{I}_{\mathrm{V}}}\right|=\left|\beta_{4} \mathrm{~S}_{\mathrm{H}}\right|,\left|\frac{\partial \mathrm{f}_{2}}{\partial \mathrm{R}_{\mathrm{H}}}\right|=\left|\frac{\partial \mathrm{f}_{2}}{\partial \mathrm{S}_{\mathrm{V}}}\right|=\left|\frac{\partial \mathrm{f}_{2}}{\partial \mathrm{E}_{\mathrm{V}}}\right|=0<\infty, \\
& \left|\frac{\partial \mathrm{f}_{3}}{\partial \mathrm{E}_{\mathrm{H}}}\right|=|\mathrm{a} \alpha|,\left|\frac{\partial \mathrm{f}_{3}}{\partial \mathrm{I}_{\mathrm{HA}}}\right|=\left|-\left(\delta+\mu_{\mathrm{H}}+\omega_{1}\right)\right|<\infty, \\
& \left|\frac{\partial \mathrm{f}_{3}}{\partial \mathrm{S}_{\mathrm{H}}}\right|=\left|\frac{\partial \mathrm{f}_{3}}{\partial \mathrm{I}_{\mathrm{HA}}}\right|=\left|\frac{\partial \mathrm{f}_{3}}{\partial \mathrm{I}_{\mathrm{HQ}}}\right|=\left|\frac{\partial \mathrm{f}_{3}}{\partial \mathrm{R}_{\mathrm{H}}}\right|=\left|\frac{\partial \mathrm{f}_{3}}{\partial \mathrm{S}_{\mathrm{V}}}\right|=\left|\frac{\partial \mathrm{f}_{3}}{\partial \mathrm{E}_{\mathrm{V}}}\right|=\left|\frac{\partial \mathrm{f}_{3}}{\partial \mathrm{I}_{\mathrm{V}}}\right|=0<\infty \text {. } \\
& \left|\frac{\partial \mathrm{f}_{4}}{\partial \mathrm{E}_{\mathrm{H}}}\right|=|(1-\mathrm{a}) \alpha|,\left|\frac{\mathrm{df}_{4}}{\partial \mathrm{I}_{\mathrm{HS}}}\right|=\left|-\left(\delta+\mu_{\mathrm{H}}+\omega_{2}\right)\right|<\infty, \\
& \left|\frac{\partial \mathrm{f}_{4}}{\partial \mathrm{S}_{\mathrm{H}}}\right|=\left|\frac{\partial \mathrm{f}_{4}}{\partial \mathrm{I}_{\mathrm{HA}}}\right|=\left|\frac{\partial \mathrm{f}_{4}}{\partial \mathrm{I}_{\mathrm{HQ}}}\right|=\left|\frac{\partial \mathrm{f}_{4}}{\partial \mathrm{R}_{\mathrm{H}}}\right|=\left|\frac{\partial \mathrm{f}_{4}}{\partial \mathrm{S}_{\mathrm{V}}}\right|=\left|\frac{\partial \mathrm{f}_{4}}{\partial \mathrm{E}_{\mathrm{V}}}\right|=\left|\frac{\partial \mathrm{f}_{4}}{\partial \mathrm{I}_{\mathrm{V}}}\right|=0<\infty . \\
& \left|\frac{\partial \mathrm{f}_{5}}{\partial \mathrm{I}_{\mathrm{HA}}}\right|=|\delta| \cdot\left|\frac{\partial \mathrm{f}_{5}}{\partial \mathrm{I}_{\mathrm{HS}}}\right|=|\delta|,\left|\frac{\partial \mathrm{f}_{5}}{\partial \mathrm{I}_{\mathrm{HQ}}}\right|=\left|-\left(\mu_{\mathrm{H}}+\eta\right)\right|<\infty, \\
& \left|\frac{\partial \mathrm{f}_{5}}{\partial \mathrm{S}_{\mathrm{H}}}\right|=\left|\frac{\partial \mathrm{f}_{5}}{\partial \mathrm{E}_{\mathrm{H}}}\right|=\left|\frac{\partial \mathrm{f}_{5}}{\partial \mathrm{R}_{\mathrm{H}}}\right|=\left|\frac{\partial \mathrm{f}_{5}}{\partial \mathrm{S}_{\mathrm{V}}}\right|=\left|\frac{\partial \mathrm{f}_{5}}{\partial \mathrm{E}_{\mathrm{V}}}\right|=\left|\frac{\partial \mathrm{f}_{5}}{\partial \mathrm{I}_{\mathrm{V}}}\right|=0<\infty \text {. } \\
& \left|\frac{\partial \mathrm{f}_{6}}{\partial \mathrm{I}_{\mathrm{HQ}}}\right|=|\eta|,\left|\frac{\partial \mathrm{f}_{6}}{\partial \mathrm{R}_{\mathrm{H}}}\right|=\left|-\mu_{\mathrm{H}}\right|<\infty, \\
& \left|\frac{\partial \mathrm{f}_{6}}{\partial \mathrm{S}_{\mathrm{H}}}\right|=\left|\frac{\partial \mathrm{f}_{6}}{\partial \mathrm{E}_{\mathrm{H}}}\right|=\left|\frac{\partial \mathrm{f}_{6}}{\partial \mathrm{I}_{\mathrm{HA}}}\right|=\left|\frac{\partial \mathrm{f}_{6}}{\partial \mathrm{I}_{\mathrm{HS}}}\right|=\left|\frac{\partial \mathrm{f}_{6}}{\partial \mathrm{S}_{\mathrm{V}}}\right|=\left|\frac{\partial \mathrm{f}_{6}}{\partial \mathrm{E}_{\mathrm{V}}}\right|=\left|\frac{\partial \mathrm{f}_{6}}{\partial \mathrm{I}_{\mathrm{V}}}\right|=0<\infty \\
& \left|\frac{\partial \mathrm{f}_{7}}{\partial \mathrm{S}_{\mathrm{V}}}\right|=\left|-\left(\beta_{4} \mathrm{I}_{\mathrm{V}}+\mu_{\mathrm{V}}+\varphi_{\mathrm{V}}\right)\right|<\infty,\left|\frac{\partial \mathrm{f}_{7}}{\partial \mathrm{I}_{\mathrm{V}}}\right|=-\left|\beta_{4} \mathrm{~S}_{\mathrm{V}}\right| \text {, } \\
& \left|\frac{\partial \mathrm{f}_{7}}{\partial \mathrm{S}_{\mathrm{H}}}\right|=\left|\frac{\partial \mathrm{f}_{7}}{\partial \mathrm{E}_{\mathrm{H}}}\right|=\left|\frac{\partial \mathrm{f}_{7}}{\partial \mathrm{I}_{\mathrm{HA}}}\right|=\left|\frac{\partial \mathrm{f}_{7}}{\partial \mathrm{I}_{\mathrm{HS}}}\right|=\left|\frac{\partial \mathrm{f}_{7}}{\partial \mathrm{I}_{\mathrm{HQ}}}\right|=\left|\frac{\partial \mathrm{f}_{7}}{\partial \mathrm{R}_{\mathrm{H}}}\right|=\left|\frac{\partial \mathrm{f}_{7}}{\partial \mathrm{E}_{\mathrm{V}}}\right|=0<\infty, \\
& \left|\frac{\partial \mathrm{f}_{8}}{\partial \mathrm{S}_{\mathrm{V}}}\right|=\left|\beta_{4} \mathrm{I}_{\mathrm{V}}\right|,\left|\frac{\partial \mathrm{f}_{8}}{\partial \mathrm{E}_{\mathrm{V}}}\right|=\left|-\left(\gamma_{\mathrm{V}}+\mu_{\mathrm{V}}+\varphi_{\mathrm{V}}\right)\right|<\infty,\left|\frac{\partial \mathrm{f}_{8}}{\partial \mathrm{I}_{\mathrm{V}}}\right|=\left|\beta_{4} \mathrm{~S}_{\mathrm{V}}\right|, \\
& \left|\frac{\partial \mathrm{f}_{8}}{\partial \mathrm{S}_{\mathrm{H}}}\right|=\left|\frac{\partial \mathrm{f}_{8}}{\partial \mathrm{E}_{\mathrm{H}}}\right|=\left|\frac{\partial \mathrm{f}_{8}}{\partial \mathrm{I}_{\mathrm{HA}}}\right|=\left|\frac{\partial \mathrm{f}_{8}}{\partial \mathrm{I}_{\mathrm{HS}}}\right|=\left|\frac{\partial \mathrm{f}_{8}}{\partial \mathrm{I}_{\mathrm{HQ}}}\right|=\left|\frac{\partial \mathrm{f}_{8}}{\partial \mathrm{R}_{\mathrm{H}}}\right|=0<\infty . \\
& \left|\frac{\partial \mathrm{f}_{9}}{\partial \mathrm{E}_{\mathrm{V}}}\right|=\left|\gamma_{\mathrm{V}}\right|,\left|\frac{\partial \mathrm{f}_{9}}{\partial \mathrm{I}_{\mathrm{V}}}\right|=\left|-\left(\mu_{\mathrm{V}}+\varphi_{\mathrm{V}}\right)\right|<\infty, \\
& \left|\frac{\partial \mathrm{f}_{9}}{\partial \mathrm{S}_{\mathrm{H}}}\right|=\left|\frac{\partial \mathrm{f}_{9}}{\partial \mathrm{E}_{\mathrm{H}}}\right|=\left|\frac{\partial \mathrm{f}_{9}}{\partial \mathrm{I}_{\mathrm{HA}}}\right|=\left|\frac{\partial \mathrm{f}_{9}}{\partial \mathrm{I}_{\mathrm{HS}}}\right|=\left|\frac{\partial \mathrm{f}_{9}}{\partial \mathrm{I}_{\mathrm{HQ}}}\right|=\left|\frac{\partial \mathrm{f}_{9}}{\partial \mathrm{R}_{\mathrm{H}}}\right|=\left|\frac{\partial \mathrm{f}_{9}}{\partial \mathrm{S}_{\mathrm{V}}}\right|=0<\infty . \\
& \mathrm{a} \alpha \mathrm{E}_{\mathrm{H}}-\left(\delta+\mu_{\mathrm{H}}+\omega_{1}\right) \mathrm{I}_{\mathrm{HA}}=0 \\
& (\mathrm{I}-\mathrm{a}) \alpha \mathrm{E}_{\mathrm{H}}-\left(\delta+\mu_{\mathrm{H}}+\omega_{2}\right) \mathrm{I}_{\mathrm{HS}}=0 \\
& \left(\mathrm{I}_{\mathrm{HA}}+\mathrm{I}_{\mathrm{HS}}\right) \delta-\mu_{\mathrm{H}} \mathrm{I}_{\mathrm{HQ}}=0 \\
& \eta I_{\mathrm{HQ}}-\mu_{\mathrm{H}} \mathrm{R}_{\mathrm{H}}=0 \\
& \Lambda_{\mathrm{V}}-\left(\beta_{4} \mathrm{I}_{\mathrm{V}}+\mu_{\mathrm{V}}+\varphi_{\mathrm{V}}\right) \mathrm{S}_{\mathrm{V}}=0 \\
& \beta_{4} \mathrm{I}_{\mathrm{V}} \mathrm{S}_{\mathrm{V}}-\left(\gamma_{\mathrm{V}}+\mu_{\mathrm{V}}+\varphi_{\mathrm{V}}\right) \mathrm{E}_{\mathrm{V}}=0 \\
& \gamma_{\mathrm{V}} \mathrm{E}_{\mathrm{V}}-\left(\mu_{\mathrm{H}}+\varphi_{\mathrm{H}}\right) \mathrm{I}_{\mathrm{V}}=0
\end{aligned}
$$

This shows that the partial derivatives are continuous and bounded, so that theorem (2) holds, there exists a unique solution of (1.1)-(1.9) in the region D.

\subsection{Disease Free-Equilibrium Analysis.}

To perform the disease free - equilibrium analysis of the model and to understand better the dynamics of the disease, we set each of the derived equations to equate zero and solve for $\mathrm{S}_{\mathrm{H}}, \mathrm{E}_{\mathrm{H}}, \mathrm{I}_{\mathrm{HA}}, \mathrm{I}_{\mathrm{HS}}, \mathrm{I}_{\mathrm{HQ}}, \mathrm{R}_{\mathrm{H}}, \mathrm{S}_{\mathrm{V}}, \mathrm{E}_{\mathrm{V}}$ and $\mathrm{I}_{\mathrm{V}}$. We will get fixed points for each compartment for which the system will no longer change. Solving the model equations to obtain this state, we have:

$$
\Lambda_{\mathrm{H}}-\beta_{1} \mathrm{I}_{\mathrm{HA}} \mathrm{S}_{\mathrm{H}}-\beta_{2} \mathrm{I}_{\mathrm{HS}} \mathrm{S}_{\mathrm{H}}-\beta_{3} \mathrm{I}_{\mathrm{HQ}} \mathrm{S}_{\mathrm{H}}-\beta_{4} \mathrm{I}_{\mathrm{V}} \mathrm{S}_{\mathrm{H}}-\mu_{\mathrm{H}} \mathrm{S}_{\mathrm{H}}=0
$$

$\beta_{1} \mathrm{I}_{\mathrm{HA}} \mathrm{S}_{\mathrm{H}}+\beta_{2} \mathrm{I}_{\mathrm{HS}} \mathrm{S}_{\mathrm{H}}+\beta_{3} \mathrm{I}_{\mathrm{HQ}} \mathrm{S}_{\mathrm{H}}+\beta_{4} \mathrm{I}_{\mathrm{V}} \mathrm{S}_{\mathrm{H}}-\left(\alpha+\mu_{\mathrm{H}}\right) \mathrm{E}_{\mathrm{H}}=0(16)$
Solving equations (3.1)-(3.9) simultaneously we obtain:

$$
\mathrm{E}_{0}=\left(\mathrm{S}_{\mathrm{H}}, \mathrm{E}_{\mathrm{H}}, \mathrm{I}_{\mathrm{HA}}, \mathrm{I}_{\mathrm{HS}}, \mathrm{I}_{\mathrm{HQ}}, \mathrm{R}_{\mathrm{H}}, \mathrm{S}_{\mathrm{V}}, \mathrm{E}_{\mathrm{V}}, \mathrm{I}_{\mathrm{V}}\right)=
$$




$$
\left(\frac{\Lambda_{\mathrm{H}}}{\mu_{\mathrm{H}}}, 0,0,0,0,0, \frac{\Lambda_{\mathrm{V}}}{\left(\mu_{\mathrm{V}}+\varphi_{\mathrm{V}}\right)}, 0,0\right)
$$

as the disease free - equilibrium state.

\subsection{Basic Reproduction Ratio}

The basic reproductive ratio (number) $\mathrm{R}_{0}$ is used in determining the transmission capability of the disease. It is taken to be the average number of secondary diseases delivered by a primary case of an infection in a host population that is completely susceptible. $\mathrm{R}_{0}$ is a threshold parameter that decides if a disease can strive in a population. If $\mathrm{R}_{0}<1$, then the disease will die out with time in the population. If not, it will lead to endemic state in the population.

We use the next generation operator to compute the reproductive ratio $\mathrm{R}_{0}=\mathrm{FV}^{-1}$ which gives the

rate at which individuals in compartment $j$ generate new infections in compartment $\mathrm{i}$ times average length of time individuals spend in single visit to compartment $\mathrm{j}$. Let $\mathrm{F}$ be defined as the Jacobian of $f_{i}$ where $f_{i}$ is the rate of appearance of new infection in the compartment $i$ and $V$ is defined as the Jacobian of $v_{j}$ where $v_{j}$ is the rate of transfer of infections from infected compartment to another compartment $\mathrm{j}$, all represented in a matrix form.

$$
\begin{gathered}
\mathrm{f}=\left(\begin{array}{c}
\text { Define } \mathrm{f}_{\mathrm{i}}=\left(\mathrm{E}_{\mathrm{H}}, \mathrm{I}_{\mathrm{HA}}, \mathrm{I}_{\mathrm{HS}}, \mathrm{I}_{\mathrm{HQ}}, \mathrm{E}_{\mathrm{V}}, \mathrm{I}_{\mathrm{V}}\right) \\
\beta_{1} \mathrm{I}_{\mathrm{HA}} \mathrm{S}_{\mathrm{H}}+\beta_{2} \mathrm{I}_{\mathrm{HS}} \mathrm{S}_{\mathrm{H}}+\beta_{3} \mathrm{I}_{\mathrm{HQ}} \mathrm{S}_{\mathrm{H}}+\beta_{4} \mathrm{I}_{\mathrm{V}} \mathrm{S}_{\mathrm{H}} \\
0 \\
0 \\
0 \\
\beta_{4} \mathrm{I}_{\mathrm{V}} \mathrm{S}_{\mathrm{V}} \\
0 \\
-\left(\alpha+\mu_{\mathrm{H}}\right) \mathrm{E}_{\mathrm{H}} \\
\mathrm{v}=\left(\begin{array}{c}
-\mathrm{a} \alpha \mathrm{E}_{\mathrm{H}}+\left(\delta+\mu_{\mathrm{H}}+\omega_{1}\right) \mathrm{I}_{\mathrm{HA}} \\
-(1-\mathrm{a}) \alpha \mathrm{E}_{\mathrm{H}}+\left(\delta+\mu_{\mathrm{H}}+\omega_{2}\right) \mathrm{I}_{\mathrm{HS}} \\
-\left(\mathrm{I}_{\mathrm{HA}}+\mathrm{I}_{\mathrm{HS}}\right) \delta+\left(\mu_{\mathrm{H}}+\eta\right) \mathrm{I}_{\mathrm{HQ}} \\
\left(\gamma_{\mathrm{V}}+\mu_{\mathrm{V}}+\varphi_{\mathrm{V}}\right) \mathrm{E}_{\mathrm{V}} \\
-\gamma_{\mathrm{V}} \mathrm{E}_{\mathrm{V}}+\left(\mu_{\mathrm{V}}+\varphi_{\mathrm{V}}\right) \mathrm{I}_{\mathrm{V}}
\end{array}\right)
\end{array}\right.
\end{gathered}
$$

Taking the partial derivatives of $\mathrm{f}$ and $\mathrm{v}$ at disease-free equilibrium state, we obtain the following for $\mathrm{f}$ and $\mathrm{v}$ respectively, represented as:

$$
\mathrm{F}=\left(\begin{array}{cccccc}
m_{11} & 0 & m_{13} & m_{14} & 0 & m_{16} \\
0 & 0 & 0 & 0 & 0 & 0 \\
0 & 0 & 0 & 0 & 0 & 0 \\
0 & 0 & 0 & 0 & 0 & 0 \\
0 & 0 & 0 & 0 & 0 & m_{55} \\
0 & 0 & 0 & 0 & 0 & 0
\end{array}\right)
$$

Where $m_{11}=\frac{\beta_{1} \wedge_{\mathrm{H}}}{\mu_{\mathrm{H}}}, \mathrm{m}_{13}=\frac{\beta_{2} \wedge_{\mathrm{H}}}{\mu_{\mathrm{H}}}, \mathrm{m}_{14}=\frac{\beta_{3} \wedge_{\mathrm{H}}}{\mu_{\mathrm{H}}}, \mathrm{m}_{16}=\frac{\beta_{4} \wedge_{\mathrm{H}}}{\mu_{\mathrm{H}}}, \mathrm{m}_{55}=\frac{\beta_{4} \wedge_{\mathrm{V}}}{\mu_{\mathrm{V}}+\varphi_{\mathrm{V}}}$.

And also $\mathrm{V}=\left(\begin{array}{cccccc}-a_{11} & 0 & 0 & 0 & 0 & 0 \\ -a_{21} & a_{22} & 0 & 0 & 0 & 0 \\ -a_{31} & 0 & a_{33} & 0 & 0 & 0 \\ 0 & -a_{42} & -a_{43} & a_{44} & 0 & 0 \\ 0 & 0 & 0 & 0 & a_{55} & 0 \\ 0 & 0 & 0 & 0 & -a_{65} & a_{66}\end{array}\right)$

Where $\mathrm{a}_{11}=-\left(\alpha+\mu_{\mathrm{H}}\right), \mathrm{a}_{21}=-\mathrm{a} \alpha, \mathrm{a}_{22}=\left(\delta+\mu_{\mathrm{H}}+\omega_{1}\right), \mathrm{a}_{31}=-(1-\mathrm{a}) \alpha, \mathrm{a}_{33}=\left(\delta+\mu_{\mathrm{H}}+\omega_{2}\right)$,

$$
\mathrm{a}_{42}=-\delta, \mathrm{a}_{43}=-\delta, \mathrm{a}_{44}=\left(\mu_{\mathrm{H}}+\eta\right), \mathrm{a}_{55}=\left(\gamma_{\mathrm{V}}+\mu_{\mathrm{V}}+\varphi_{\mathrm{V}}\right), \mathrm{a}_{65}=\gamma_{\mathrm{V}}, \mathrm{a}_{66}=\left(\mu_{\mathrm{V}}+\varphi_{\mathrm{V}}\right) .
$$

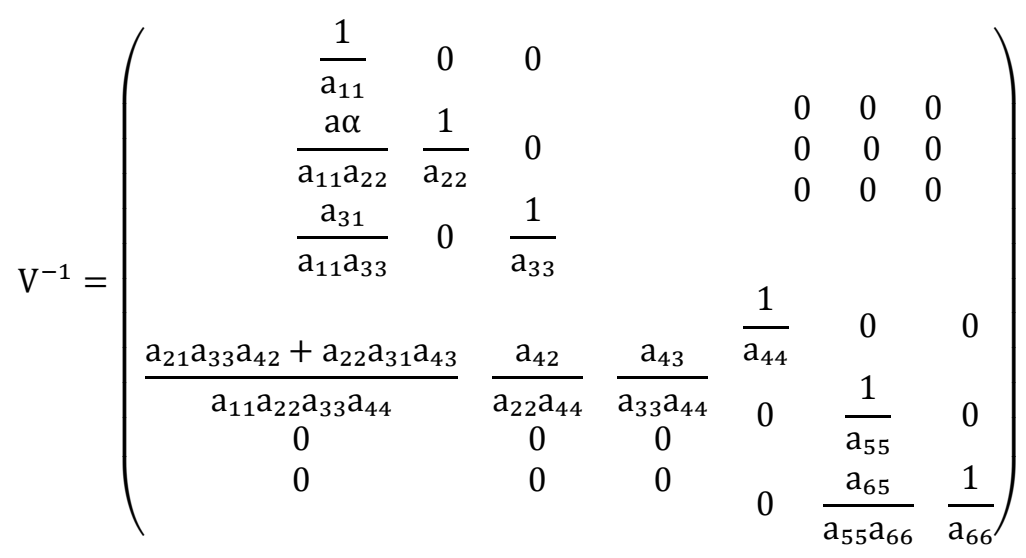




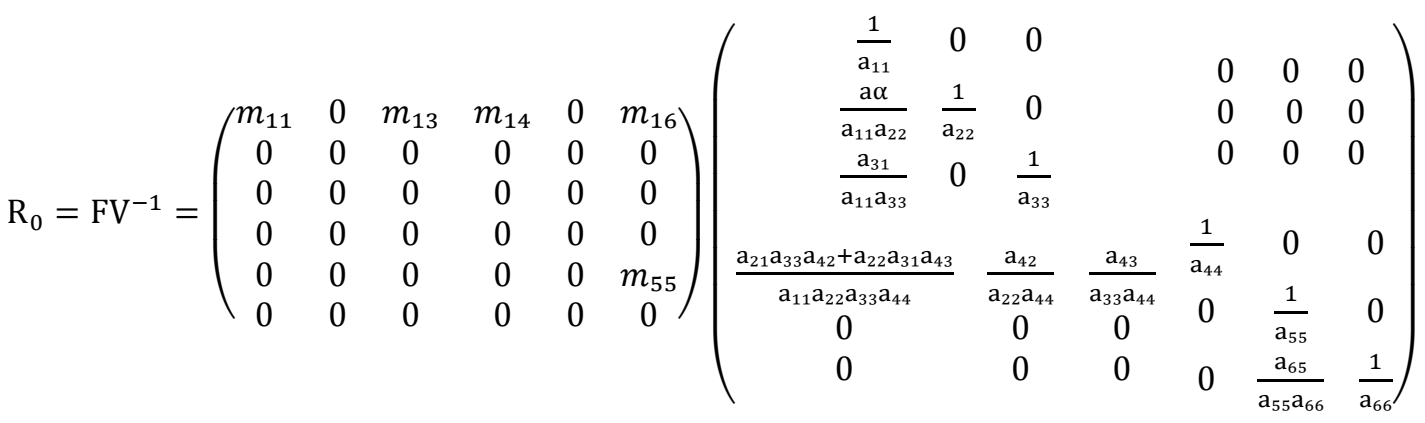

$$
\begin{aligned}
& R_{0}=\frac{\beta_{4} \Lambda_{\mathrm{V}}}{\left(\mu_{\mathrm{V}}+\varphi_{\mathrm{V}}\right)\left(\gamma_{\mathrm{V}}+\mu_{\mathrm{V}}+\varphi_{\mathrm{V}}\right)}
\end{aligned}
$$

\subsection{Stability Analysis of Disease Free-Equilibrium State}

In stability of the model, we look at the behavior of the population near the equilibrium state. The Jacobian matrix of the system of equations (1.1)-(1.9) at equilibrium $\mathrm{E}_{0}$ is given by

$$
: J E_{0}=\left(\begin{array}{ccccccccc}
-\mu_{H} & 0 & \frac{-\left(\beta_{1} \Lambda_{H}\right)}{\mu_{H}} & \frac{-\left(\beta_{2} \Lambda_{H}\right)}{\mu_{H}} & \frac{-\left(\beta_{3} \Lambda_{H}\right)}{\mu_{H}} & 0 & 0 & 0 & \frac{-\beta_{4} \Lambda_{V}}{\left(\mu_{V}+\varphi_{V}\right)} \\
\mu_{H} & -\left(\alpha+\mu_{H}\right) & 0 & 0 & 0 & 0 & 0 & 0 & 0 \\
0 & a \alpha & -\left(\delta+\mu_{H}+\omega_{1}\right) & 0 & 0 & 0 & 0 & 0 & 0 \\
0 & (1-a) \alpha & 0 & -\left(\delta+\mu_{H}+\omega_{2}\right) & 0 & 0 & 0 & 0 & 0 \\
0 & 0 & \delta & \delta & -\left(\mu_{H}+\eta\right) & 0 & 0 & 0 & 0 \\
0 & 0 & 0 & 0 & \eta & -\mu_{H} & 0 & 0 & 0 \\
0 & 0 & 0 & 0 & 0 & 0 & -\left(\beta_{4} I_{V}+\mu_{V}+\varphi_{V}\right) & 0 & 0 \\
0 & 0 & 0 & 0 & 0 & 0 & \beta_{4} I_{V} & -\left(\gamma_{V}+\mu_{V}+\varphi_{V}\right) & 0 \\
0 & 0 & 0 & 0 & 0 & 0 & 0 & \gamma_{V} & -\left(\mu_{V}+\varphi_{V}\right)
\end{array}\right)
$$

Using elementary row transform on the following matrix below:

$$
\left(\begin{array}{cccccccc}
-\mathrm{a}_{1} & 0 & -\mathrm{a}_{2} & -\mathrm{a}_{3} & -\mathrm{a}_{4} & 0 & 0 & -\mathrm{a}_{5} \\
\mathrm{~b}_{1} & -\mathrm{b}_{2} & 0 & 0 & 0 & 0 & 0 & 0 \\
0 & \mathrm{c}_{1} & -\mathrm{c}_{2} & 0 & 0 & 0 & 0 & 0 \\
0 & \mathrm{~d}_{1} & 0 & -\mathrm{d}_{2} & 0 & 0 & 0 & 0 \\
0 & 0 & \mathrm{e}_{1} & \mathrm{e}_{2} & -\mathrm{e}_{3} & 0 & 0 & 0 \\
0 & 0 & 0 & 0 & 0 & -\mathrm{g}_{1} & 0 & 0 \\
0 & 0 & 0 & 0 & 0 & \mathrm{~h}_{1} & -\mathrm{h}_{2} & 0 \\
0 & 0 & 0 & 0 & 0 & 0 & \mathrm{j}_{1} & -\mathrm{j}_{2}
\end{array}\right)
$$

We obtain

$$
\left(\begin{array}{cccccccc}
-\mathrm{k}_{1} & 0 & 0 & 0 & 0 & 0 & 0 & 0 \\
\mathrm{~b}_{1} & -\mathrm{b}_{2} & 0 & 0 & 0 & 0 & 0 & 0 \\
0 & \mathrm{c}_{1} & -\mathrm{c}_{2} & 0 & 0 & 0 & 0 & 0 \\
0 & \mathrm{~d}_{1} & 0 & -\mathrm{d}_{2} & 0 & 0 & 0 & 0 \\
0 & 0 & \mathrm{e}_{1} & \mathrm{e}_{2} & -\mathrm{e}_{3} & 0 & 0 & 0 \\
0 & 0 & 0 & 0 & 0 & -\mathrm{g}_{1} & 0 & 0 \\
0 & 0 & 0 & 0 & 0 & \mathrm{~h}_{1} & -\mathrm{h}_{2} & 0 \\
0 & 0 & 0 & 0 & 0 & 0 & \mathrm{j}_{1} & -\mathrm{j}_{2}
\end{array}\right)
$$

Where

$$
\begin{gathered}
\mathrm{a}_{1}=-\mu_{\mathrm{H}}, \mathrm{a}_{2}=\frac{-\left(\beta_{1} \Lambda_{\mathrm{H}}\right)}{\mu_{\mathrm{H}}}, \mathrm{a}_{3}=\frac{-\left(\beta_{2} \Lambda_{\mathrm{H}}\right)}{\mu_{\mathrm{H}}}, \mathrm{a}_{4}=\frac{-\left(\beta_{3} \Lambda_{\mathrm{H}}\right)}{\mu_{\mathrm{H}}}, \mathrm{a}_{5}=\frac{-\left(\beta_{4} \Lambda_{\mathrm{H}}\right)}{\mu_{\mathrm{H}}} \\
b_{1}=\mu_{\mathrm{H}}, \mathrm{b}_{2}=-\left(\alpha+\mu_{\mathrm{H}}\right), \\
\mathrm{c}_{1}=\mathrm{a} \alpha, \mathrm{c}_{2}=-\left(\delta+\mu_{\mathrm{H}}+\omega_{1}\right), \mathrm{d}_{1}=(1-\mathrm{a}) \alpha, \mathrm{d}_{2}=-\left(\delta+\mu_{\mathrm{H}}+\omega_{2}\right), \mathrm{e}_{1}=\delta, \mathrm{e}_{2}=\delta, \mathrm{e}_{3}=-\left(\mu_{\mathrm{H}}+\eta\right) \\
\mathrm{g}_{1}=-\left(\beta_{4} \mathrm{I}_{\mathrm{V}}+\mu_{\mathrm{V}}+\varphi_{\mathrm{V}}\right), \mathrm{h}_{1}=\beta_{4} \mathrm{I}_{\mathrm{V}}, \mathrm{h}_{2}=\left(\gamma_{\mathrm{V}}+\mu_{\mathrm{V}}+\varphi_{\mathrm{V}}\right), \mathrm{j}_{1}=\gamma_{\mathrm{V}}, \mathrm{j}_{2}=-\left(\mu_{\mathrm{V}}+\varphi_{\mathrm{V}}\right) .
\end{gathered}
$$




$$
\mathrm{k}_{1}=-\mathrm{a}_{1}-\left(\mathrm{b}_{1} *\left(\frac{\mathrm{c}_{1} *\left(\mathrm{a}_{2}-\frac{\mathrm{a}_{4} * \mathrm{e}_{1}}{\mathrm{e}_{3}}\right)}{\mathrm{c}_{2}}-\frac{\mathrm{d}_{1} *\left(\mathrm{a}_{3}+\frac{\mathrm{a}_{4} * \mathrm{e}_{2}}{\mathrm{e}_{3}}\right)}{\mathrm{d}_{2}}\right)\right) / \mathrm{b}_{2} .
$$

Hence the eigenvalues are:

$$
\lambda_{1}=\mathrm{k}_{1}, \lambda_{2}=\mathrm{b}_{2}, \lambda_{3}=\mathrm{c}_{2}, \lambda_{4}=\mathrm{d}_{2}, \lambda_{5}=\mathrm{e}_{3}, \lambda_{6}=\mathrm{g}_{1}, \lambda_{7}=\mathrm{h}_{2} \text { and } \lambda_{8}=\mathrm{j}_{2} .
$$

When $\lambda_{\mathrm{i}} \leq 0$ for all $\mathrm{i}=1,2, \ldots, 8$ the system is said to be locally asymptotically stable at disease-free equilibrium. Since all the eigenvalues have negative real paths, we say that $\mathrm{R}_{0}<1$ showing locally asymptotically stability which ends the proof. The disease gradually dies out at this point.

\subsection{Global Stability State for the Disease-Free Equilibrium}

The global asymptotic stability of the disease-free state of the model is investigated using the theorem by CastilloChavez et al (2002). The model is written into the form:

$$
\begin{gathered}
\frac{d X}{d t}=F(X, I) \\
\frac{d I}{d t}=G(X, I), G(X, 0)=0
\end{gathered}
$$

Where $\mathrm{X} \in \mathrm{R}^{\mathrm{m}}$ denotes (its components) the number of uninfected individuals and $\mathrm{I} \in \mathrm{R}^{\mathrm{n}}$ denotes (its components) the number of infected individuals including latent, infectious etc. $E_{0}=\left(X^{*}, 0\right)$ denotes the disease-free equilibrium of this system.

The conditions (H1) and (H2) below must be met to guarantee local asymptotic stability.

(H1) $\frac{d X}{d t}=F(X, 0), X^{*}$ is globally asymptotically stable.
(H2) $\mathrm{G}(\mathrm{X}, \mathrm{I})=\mathrm{AI}-\hat{\mathrm{G}}(\mathrm{X}, \mathrm{I}), \hat{\mathrm{G}}(\mathrm{X}, \mathrm{I}) \geq 0$ for $(\mathrm{X}, \mathrm{I}) \in \Omega$.

Where $A=\frac{\partial G}{\partial \mathrm{I}}\left(X^{*}, 0\right)$ is an $M$-matrix (the off diagonal elements of $A$ are nonnegative) and $\Omega$ is the region where the model makes biological sense.

Then the disease- free equilibrium $\mathrm{E}_{0}=\left(\mathrm{X}^{*}, 0\right)$ is globally asymptotically stable provided that $R_{0} \leq 1$.

$$
\begin{gathered}
\text { LetX }=\left(S_{H}, R_{H}, S_{V}\right), I=\left(E_{H}, I_{H A}, I_{H S}, I_{H Q}, E_{V}, I_{V}\right) . \\
F(X, 0)=\left(\begin{array}{c}
\Lambda_{H}-\mu_{H} S_{H} \\
\mu_{H} R_{H} \\
\Lambda_{V}-\left(\mu_{V}+\varphi_{V}\right) S_{V}
\end{array}\right)
\end{gathered}
$$

The equations are linear and its solution can be easily found as $\mathrm{R}_{\mathrm{H}}(\mathrm{t})=\mathrm{R}(0) \mathrm{e}^{\mu_{\mathrm{H}(\mathrm{t})}}$,

$$
\begin{gathered}
\mathrm{S}_{\mathrm{H}}(\mathrm{t})=\left(\mathrm{S}_{\mathrm{H}}(0)\right) \mathrm{e}^{\frac{\Lambda_{H}}{\mu_{H}}(t)} \text { and } \mathrm{S}_{\mathrm{V}}(\mathrm{t})=\left(\mathrm{S}_{\mathrm{V}}(0)\right) e^{\frac{\Lambda_{\mathrm{V}}}{\left(\mu_{\mathrm{V}}+\varphi_{\mathrm{V}}\right)}(t)} . \\
\text { Clearly, } \mathrm{R}_{\mathrm{H}}(\mathrm{t}) \rightarrow 0, \mathrm{~S}_{\mathrm{H}}(\mathrm{t}) \rightarrow \mathrm{N}_{\mathrm{H}} \text { as } \mathrm{t} \rightarrow \infty \text { and } \mathrm{S}_{\mathrm{V}}(\mathrm{t}) \rightarrow \\
\mathrm{N}_{\mathrm{V}} \text { as } \mathrm{t} \rightarrow \infty
\end{gathered}
$$

Next we show that condition (H2) is greater than or equal to zero as follows:

$$
\begin{aligned}
& \mathrm{G}(\mathrm{X}, \mathrm{I})=\left(\begin{array}{c}
\beta_{1} \mathrm{I}_{\mathrm{HA}} \mathrm{S}_{\mathrm{H}}+\beta_{2} \mathrm{I}_{\mathrm{HS}} \mathrm{S}_{\mathrm{H}}+\beta_{3} \mathrm{I}_{\mathrm{HQ}} \mathrm{S}_{\mathrm{H}}+\beta_{4} \mathrm{I}_{\mathrm{V}} \mathrm{S}_{\mathrm{H}}-\left(\alpha+\mu_{\mathrm{H}}\right) \mathrm{E}_{\mathrm{H}} \\
\mathrm{a} \alpha \mathrm{E}_{\mathrm{H}}-\left(\delta+\mu_{\mathrm{H}}+\omega_{1}\right) \mathrm{I}_{\mathrm{HA}} \\
(1-\mathrm{a}) \alpha \mathrm{E}_{\mathrm{H}}-\left(\delta+\mu_{\mathrm{H}}+\omega_{2}\right) \mathrm{I}_{\mathrm{HS}} \\
\left(\mathrm{I}_{\mathrm{HA}}+\mathrm{I}_{\mathrm{HS}}\right) \delta-\left(\mu_{\mathrm{H}}+\eta\right) \mathrm{I}_{\mathrm{HQ}} \\
\beta_{4} \mathrm{I}_{\mathrm{V}} S_{\mathrm{V}}-\left(\gamma_{\mathrm{V}}+\mu_{\mathrm{V}}+\varphi_{\mathrm{V}}\right) \mathrm{E}_{\mathrm{V}} \\
\gamma_{\mathrm{V}} \mathrm{E}_{\mathrm{V}}-\left(\mu_{\mathrm{V}}+\varphi_{\mathrm{V}}\right) \mathrm{I}_{\mathrm{V}}
\end{array}\right) \\
& \mathrm{AI}=\left(\begin{array}{cccccc}
-\left(\alpha+\mu_{\mathrm{H}}\right) & \beta_{1} \mathrm{~S}_{\mathrm{H}} & \beta_{2} \mathrm{~S}_{\mathrm{H}} & \beta_{3} \mathrm{~S}_{\mathrm{H}} & 0 & \beta_{4} \mathrm{~S}_{\mathrm{H}} \\
\mathrm{a} \alpha & -\left(\delta+\mu_{\mathrm{H}}+\omega_{1}\right) & 0 & 0 & 0 & 0 \\
(1-\mathrm{a}) \alpha & 0 & -\left(\delta+\mu_{\mathrm{H}}+\omega_{2}\right) & 0 & 0 & 0 \\
0 & \delta & \delta & -\left(\mu_{\mathrm{H}}+\eta\right) & 0 & 0 \\
0 & 0 & 0 & 0 & -\left(\gamma_{\mathrm{V}}+\mu_{\mathrm{V}}+\varphi_{\mathrm{V}}\right) & \beta_{4} \mathrm{~S}_{\mathrm{V}} \\
0 & 0 & 0 & 0 & \gamma_{\mathrm{V}} & -\left(\mu_{\mathrm{V}}+\varphi_{\mathrm{V}}\right)
\end{array}\right)\left(\begin{array}{c}
\mathrm{E}_{\mathrm{H}} \\
\mathrm{I}_{\mathrm{HA}} \\
\mathrm{I}_{\mathrm{HS}} \\
\mathrm{I}_{\mathrm{HQ}} \\
\mathrm{E}_{\mathrm{V}} \\
\mathrm{I}_{\mathrm{V}}
\end{array}\right) \\
& \hat{\mathrm{G}}=\left(\begin{array}{c}
0+\beta_{1} \mathrm{I}_{\mathrm{HA}}\left(\frac{\Lambda_{\mathrm{H}}}{\mu_{\mathrm{H}}}-\mathrm{S}_{\mathrm{H}}\right)+\beta_{2} \mathrm{I}_{\mathrm{HS}}\left(\frac{\Lambda_{\mathrm{H}}}{\mu_{\mathrm{H}}}-\mathrm{S}_{\mathrm{H}}\right)+\beta_{3} \mathrm{I}_{\mathrm{HQ}}\left(\frac{\Lambda_{\mathrm{H}}}{\mu_{\mathrm{H}}}-\mathrm{S}_{\mathrm{H}}\right)+0+\beta_{4} \mathrm{I}_{\mathrm{V}}\left(\frac{\Lambda_{\mathrm{H}}}{\mu_{\mathrm{H}}}-\mathrm{S}_{\mathrm{H}}\right) \\
0 \\
0 \\
0 \\
\beta_{4} \mathrm{I}_{\mathrm{V}}\left(\frac{\Lambda_{\mathrm{V}}}{\left(\mu_{\mathrm{V}}+\varphi_{\mathrm{V}}\right)}-\mathrm{S}_{\mathrm{V}}\right) \\
0
\end{array}\right)
\end{aligned}
$$

If $\hat{\mathrm{G}}=(X, I)=\left(\mathrm{S}_{\mathrm{H}}^{*}>\mathrm{S}_{\mathrm{H}}\right)$ and $\left(\mathrm{S}_{\mathrm{V}}^{*}>\mathrm{S}_{\mathrm{V}}\right)$ we see that $E_{0}=X^{*}=\left(\frac{\Lambda_{H}}{\mu_{H}}, 0,0,0,0,0, \frac{\Lambda_{V}}{\mu_{V}+\varphi_{V}}, 0,0\right)$ is the global asymptotical stability of equilibrium of $\frac{d X}{d t}=F(X, 0)$. 
Table 2. Parameter, Descriptions, Values and References.

\begin{tabular}{|c|c|c|c|}
\hline Parameters & Descriptions & Values & Sources \\
\hline$\Lambda_{H}$ & Birth or Immigration rate of Susceptible human population, & 2000 & 18 \\
\hline$\Lambda_{V}$ & Birth or Immigration rate of Susceptible vector population, & 500 & 18 \\
\hline$\mu_{H}$ & Natural death rate of humans & 0.02 & CIA-2017 \\
\hline$\beta_{H}$ & Effective contact rate between infective vector or its contaminants with the Susceptible human per contact. & 0.08 & 18 \\
\hline$\beta_{v}$ & Effective contact rate between Infectious vector and Susceptible vector per contact. & 0.02 & Estimated \\
\hline$\varphi_{V}$ & Human induced control & 0.02 & 18 \\
\hline$\gamma_{V}$ & Rate at which the exposed vectors move into the infectious population. & 0.001 & Estimated \\
\hline $\mathrm{H}$ & Recovery rate of the quarantined humans & 0.25 & Estimated \\
\hline A & Proportion of exposed that are asymptomatic & 0.15 & Estimated \\
\hline$(1-a)$ & Proportion of exposed that are symptomatic & 0.15 & Estimated \\
\hline$\alpha_{H}^{-1}$ & Latency period of Lassa fever in humans days, $3-14$ & 0.5 & 19 \\
\hline$\delta_{H}^{-1}$ & Human Infectious period days, $3-5$ & 0.25 & Estimated \\
\hline$\omega_{1}$ & Lassa fever induced death rate of Asymptomatic infected humans per contact per day. & 0.00019231 & 19 \\
\hline$\omega_{2}$ & Lassa fever-induced death rate of Symptomatic infected humans per contact per day. & 0.00019231 & 19 \\
\hline
\end{tabular}

\section{Result and Discussion}

In this research work, the disease free equilibrium was analyzed. The next generation matrix method was used to calculate the reproduction number which showed that $\mathrm{R}_{0}<1$ when locally asymptotically stable, which will lead to a reduction in the spread of the disease. Using the CastilloChavez theorem, we established the global asymptotic stability of the disease-free equilibrium which indicates that the use of quarantine will help in submerging the emergence of new infectious diseases and put in serious check the hazardous movement of the infected thereby bringing to a great reduction in the continuity of the disease in the population.

Numerically the model equations using the parameter values displayed in Table 2 with the aid of mat lab clearly showed us that there exists the symptomatic and asymptomatic behavior of the virus. Although the symptomatic individuals are highly infectious, the asymptomatic individuals also contributed to the spread of the disease putting the susceptible population at a great risk. The isolation of asymptomatic and symptomatic individuals respectively on early diagnosis helped to endanger the lives of those exposed to the disease as immediate treatment is given, thereby reducing the spread of the disease if kept consistent. Hence there will be a drastic reduction on the number of the exposed individuals. A relapse in this technique could lead to a drop on the recovery rate which will lead to reinfection of the disease in the population.

\section{Conclusion}

In this work, a determistic mathematical model was formed incorporating the quarantine system as the major control measure alongside others. The analytic behaviour of the model showed that the disease-free equilibrium of the model is both locally and asymptotically stable if the reproduction number of the model is less than 1 and unstable if it is greater than 1 . From the graph, it is observed that the quarantine system has a significant effect in increasing the rate of recovering of humans down with the disease and lowering the rate of contraction of the disease. Therefore we suggest that the continuous use of quarantine system should be employed and sustained alongside other control measures for all members of the population where lassa virus is dominant.

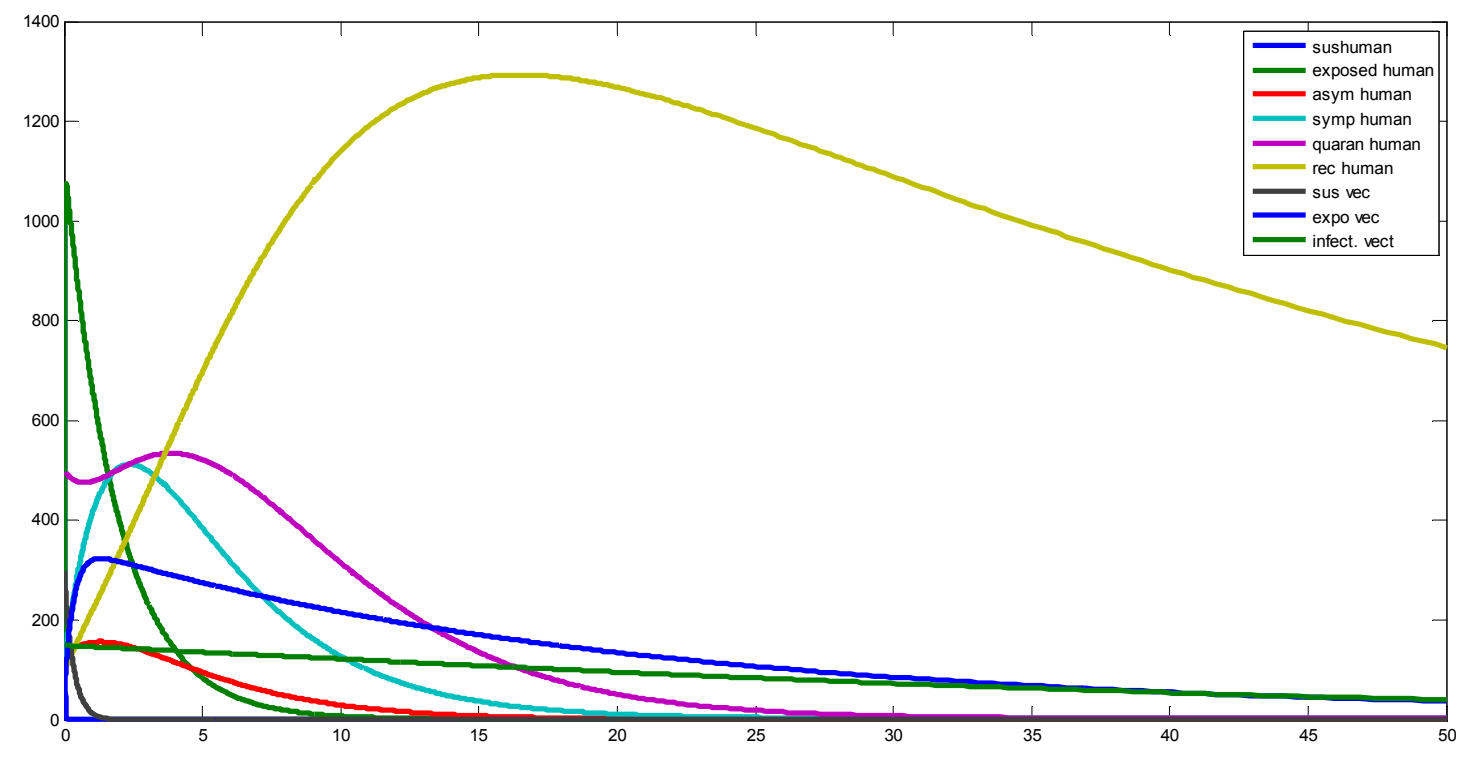

Figure 1. The plot of behavioral graph of humans on quarantining against time $t$. 


\section{References}

[1] Carey D., Kemp G, White H, Pinneo L, Addy R, Fom A, Strolcasals J, Henderson B. Lassa fever epide miological aspects of the 1970 epidemic, Jos, Nigeria. Transactions of the Royal Society of Tropical Medicine and Hygiene 1972, 66 (3): 402-408.

[2] World Health Organization (WHO) Lassa Fever outbreak in Nigeria, 2018, Available: http//www.who.int/medicalcentre, 20 April, 2018.

[3] Public Health England: lassa Fever: Origins, Reservoirs Transmission and guidelines Archived 2 February 2016 at the wayback machine. First published: 5 September, 2014 Last updated: 1 April 2016.

[4] Nwasuka C. S., Nwachukwu E. I. and Nwachukwu C. P. Mathematical model of the Transmission Dynamics of Lassa Fever with Separation of Infected individual and treatment as control measures: Journal of Advances in Mathematics and computer 9: Article No. JAMCS. 48982.

[5] Nigerian Lassa Fever Resurgence: Prevention and control; 2018. Available: http//russelsmithgroup.com/health-andsofety/Nigerian-lassa-fever-resurgence-prevention-control/. $31^{\text {st }}$ January, 2018.

[6] Amorosa V., MacNeil, A., McConnell R. et al. Imported Lassa Fever, Pennsylvania, USA; 2010. Emerging Infectious Diseases. 2010; 16 (10): 1598-600.

[7] Omalibu, S. A., Badaru, S. O., Okokhere, P., Asogun, D., Drosten, C., Emmerich, P. Lassa Fever, Nigeria 2003 and 2004. Emerging infectious Diseases, 11, 2007; 642-1644.

[8] Austo, F. B, Bewick, S. and Fagan, W. mathematical model of zika virus with vertical transmission infectious disease modeling, 2 (2); 244-267.

[9] Caminade, C., Turner, T., Metel mann, S., Hesson, J. C., Blagrove, M. S., Solomon, T. Baylis M. Global risk model for vector borne transmission of zika virus reveals the role of elnino 2015. Proceedings of the National Academy of Sciences, 2017, 114 (1); 119-124.

[10] Kernels, Solen, Koivogui, Lamine, Magassouba, N'faly,
Koulemou, Kekoure, Lewis, Rosamund., Aplogan, Aristide, Grais, F. Rebecca., Guerin, J. Philippe, Fichet Calvet, Elisabeth: prevalence and risk factors of Lassa Seropositivity in inhabitants of the forest Region of Guinea: A crosssectional study.

[11] Okuonghae, D., Okuonghae, I. A., Mathematical model for Lassa fever, Journal of National Association of Mathematical Physics, 10, 2006, 457-464.

[12] Ogabi, C. O., Olusa, T. V., Machufor, M. A., Controlling Lassa Fever in Northern part of Edo State, Nigeria using SIR model, New Science Journal, 5 (12), 2012, 115-121.

[13] Omorah, M. O., Ojo, M. S., Usman, D. J., Ademu, A., Basic reproductive Number for the spread and control of Lassa Fever, International Journal of Mathematics rends and Technology, 30 (1), 2016, 1-7.

[14] Faniran, S. T., A mathematical modeling of lassa fever dynamics with Non-drug compliance rate. International Journal of Mathematics Trends and Technology (IJMITT) volume 47 Number 5 July 2017.

[15] Modeling the effect of treatment, vaccination and public health education campaign on the transmission tuberculosis infectious. Inter disciplinary science reviews. 2019; 14 (1); $70-85$.

[16] Peter, O. J., Ibrahim M. O., Akinduko, O. B., Rabiu, M. Mathematical Model for the control of Typhoid Fever. IOSR Journal of Mathematics (IOSR-JM).e-ISSN: 2278-5728, PISSN: 2319-765X. Volume 13, Issue 4 ver. 11 (July-August, 2017), pp 60-66. www.Iosrjournals.org.

[17] Derrick, N. R. and Grossman, S. L. (1976). Differential Equation with application. Addison Wesley Publishing Company. Inc. Philippines.

[18] Mori, Y., Notomi, T. (2009). Loop-mediated Isothermal Amplification (LAMP): A Rapid, Accurate, and Cost effective Diagnostic method for infectious Diseases J. Infect. Chemosther. 15, 62-69. http://doi.org/101007/510156-0090669-9.

[19] World Health Organization, Lassa fever-Nigeria, World Health Organization, Geneva, Switzerland, 2016. 